\title{
Charged-Current Disappearance Measurements in the NuMI Off-Axis Beam
}

\author{
R H Bernstein, Fermi National Accelerator Laboratoryt
}

\begin{abstract}
This article studies the potential of combining charged-current disappearance measurements of $\nu_{\mu} \rightarrow \nu_{\tau}$ from MINOS and an off-axis beam. I find that the error on $\Delta m^{2}$ from a $100 \mathrm{kt}$-yr off-axis measurement is a few percent of itself. Further, I find little improvement to an off-axis measurement by combining it with MINOS.
\end{abstract}

Several authors have suggested that an off-axis beam can be used in combination with the MINOS measurement to measure $\Delta m^{2}$ in a NuMI off-axis beam to roughly $1 \%$ with a $100 \mathrm{kt}-y r$ exposure, e.g. $\Delta m^{2}=.0030 \pm .00030$. 11, 2] I have examined this claim with a simulation using a standard off-axis configuration, approximate detector resolutions, and the Feldman-Cousins prescription for the construction of $\Delta \chi^{2}$. I find this claim unwarranted unless we assume $\sin ^{2} 2 \theta=1$ and make optimistic assumptions concerning the errors.

\section{Physics and Detector Assumptions}

I assume an off-axis detector with $r=10 \mathrm{~km}$ off the beam axis at the FNAL/Soudan distance of $732 \mathrm{~km}$. The spectrum before is applied is shown on the left-hand side of Fig. 11 assuming no oscillations.

The spectrum is sufficiently narrow that it is instructive to consider it to be a $\delta$-function. Then there is no spectral information since all neutrinos are at the same energy, and only a total rate test can be performed. In this case neutral current contamination with low missing neutrino energy is the dominant source of background. This analysis is based on the spectral test but this argument illustrates why the background is the dominant source of error.

Based on the typical current detector designs I posit a non-magnetized detector. There is little advantage to a mangetic field because there is simply not enough lever arm to determine the muon momentum for curvature. Calorimetry is performed by hit-counting, so that the total number of hits is roughly proportional to neutrino energy. No muon tracking is attempted. Normally one would look for long tracks protruding past the end of the hadronic shower to signal the presence of a muon, but at these energies all outgoing tracks are very close in length. The error on momentum from length determination goes as a (constant term dependent upon straggling)/track length, and with the short track lengths at a $\mathrm{GeV}$ or less this is not sufficiently precise. Hence the best way to determine momentum is from counting hits as a measurement of $d E / d x$ energy deposit. Discussions with FMMF collaborators who used hit counting

† Fermi National Accelerator Laboratory, Batavia IL 60510 USA, rhbob@fnal.gov 
give resolutions typically of order $1.0 / \sqrt{E}$. [3] In what follows I will assume $55 \% / \sqrt{E}$ for the shower as a "best-possible" case.

\section{Uncertainties}

The sources of uncertainty used in this study are given in Table 1. I assume a 100 $\mathrm{kTon} \cdot \mathrm{yr}$ exposure, or 10 years of calendar time for a $10 \mathrm{kTon}$ detector. The error on the correlated flux could come from normalization of fiducial volume and extrapolation to the off-axis detector. Random flux errors are identical to those assumed by MINOS. Errors on the shape of the extrapolated spectrum come from uncertainties in FLUKA or GEANT and the precise location of beam elements. The values for the shape uncertainty are based on discussions with and work by Para and Szleper. [4]

\begin{tabular}{|c|c|c|}
\hline Statistical & 100 kton $\cdot$ years & \\
\hline \multicolumn{3}{|l|}{ Beam } \\
\hline $\begin{array}{l}\text { Correlated Flux } \\
\text { Random Flux } \\
\text { Shape }\end{array}$ & $\begin{array}{c}3 \% \\
2 \% \text { in any } 1 \mathrm{GeV} \text { bin } \\
A \sin \left(\lambda E_{\nu} / 5 .+\phi\right) \\
-.10<A<.10 \text { flat } \\
0<\lambda<2 \pi \times 5 \text { flat } \\
0<\phi<2 \pi \text { flat }\end{array}$ & $\frac{\text { See }}{\frac{\text { hep-ex/0110001, }}{0110032}}$ \\
\hline
\end{tabular}

\begin{tabular}{|l|c|l|}
\hline Detector & & \\
\hline Hadronic Energy & $0.55 / \sqrt{E}$ & \\
Muon Momentum & not separately seen & \\
& include with hadron shower energy & \\
\hline
\end{tabular}

Table 1. Sources of Uncertainty Assumed in this Analysis.

\section{Results}

The construction of the 90\% CL levels are made using the Neyman-Pearson construction, as re-invented by Feldman and Cousins. [5]; this method correctly handles the $\sin ^{2} 2 \theta=1$ physical boundary.

The results were extracted in three stages. The first used statistical and resolution errors but assumed perfect beam knowledge. The second added the effects of the assumed beam uncertainties. Finally, Fig. 2 includes these errors and the result of the statistical fluctuations of the neutral current background. I compare to the result for MINOS in Fig. 3. The errors are the standard MINOS errors and can be found in Ref. [6]. The calculations are performed in a binned $\Delta m^{2}, \sin ^{2} 2 \theta$ space and hence the bin edges are slightly irregular.

We see that the effective region at $90 \%$ CL is from $(2.80-3.20) \times 10^{-3}$ based on the off-axis data. MINOS's errors are large on this scale and contribute only a small amount; in any case some of the errors arising from the beam predictions (e.g., total flux) are correlated, so the improvement would be marginal at best. If we were to assume $\sin ^{2} 2 \theta$ is unity then a $90 \%$ CL measurement would be $2.80-3.10 \times 10^{-3}$ 

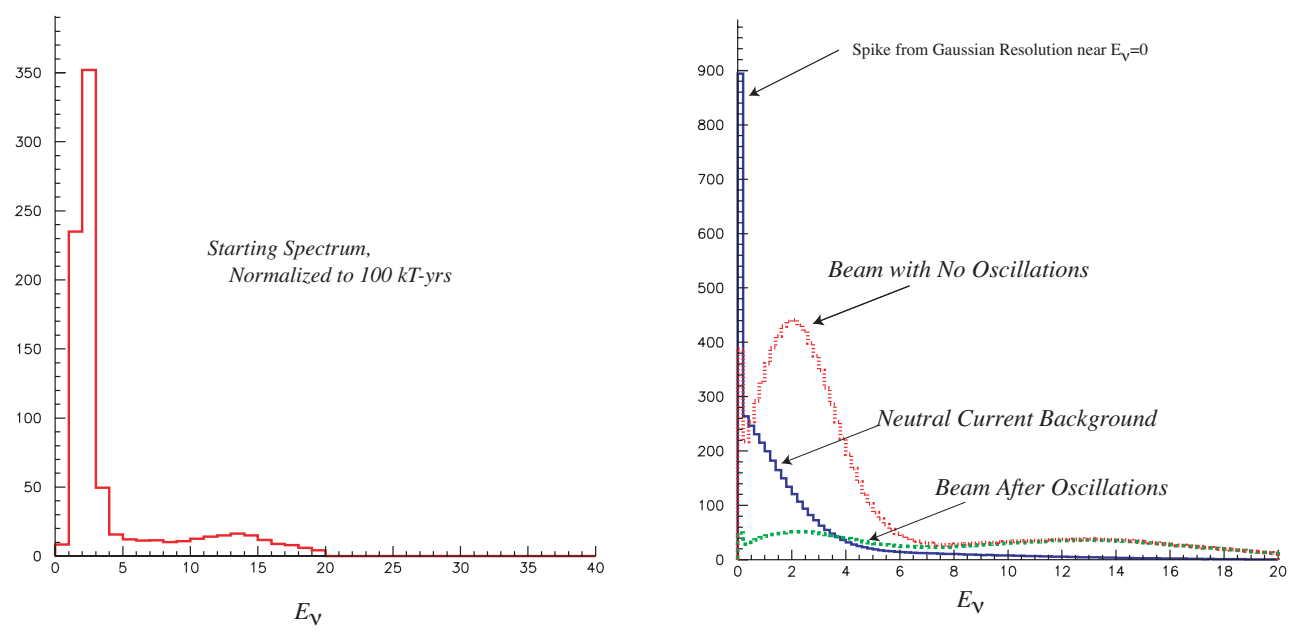

Figure 1. On the left is the spectrum for a $10 \mathrm{~km}$ off-axis detector before oscillations. On the right is the spectrum for signal and backgrounds. The spike at the origin is from neutrinos smeared to "negative" neutrino energy that I reconstruct in the zero energy bin.

and a $1 \sigma$ error would be about $0.1 \times 10^{-3}$. A $1 \%$ measurement of $\delta m^{2}$ would have an uncertainty of $\pm .03 \times 10^{-3}$, about three times smaller than this work indicates. Of course whether $\sin ^{2} 2 \theta$ is exactly unity is perhaps even more interesting than the precise value of $\Delta m^{2}$ and making such an assumption is unjustified from the data. A future paper will examine the $\nu_{\mu} \rightarrow \nu_{e}$ appearance channel including the effect of these uncertainties.

\section{References}

[1] G. Barenboim, A. de Gouvea, and M. Velasco, Neutrino Oscillations with a Proton Driver Upgrade and an Off-Axis Detector: A Case Study, hep-ph/0204208.

[2] A. Para._M.Szleper, Neutrino Oscillations Experiments using Off-axis NuMI Beam, hepex/0110032.

[3] R. Hatcher, priv. comm.

[4] M. Szleper, A. Para, Neutrino Spectrum at the Far Detector, hep-ex/0110001.

[5] Gary J. Feldman, Robert D. Cousins, A Unified Approach to the Classical Statistical Analysis of Small Signals, Phys.Rev. D57 (1998) 3873-3889

[6] R. H. Bernstein, Effects of Reconstruction Systematics with PH2L, NuMI Note SIM-0607, CC Limits and Measurements, NuMI Note SIM-0486. 


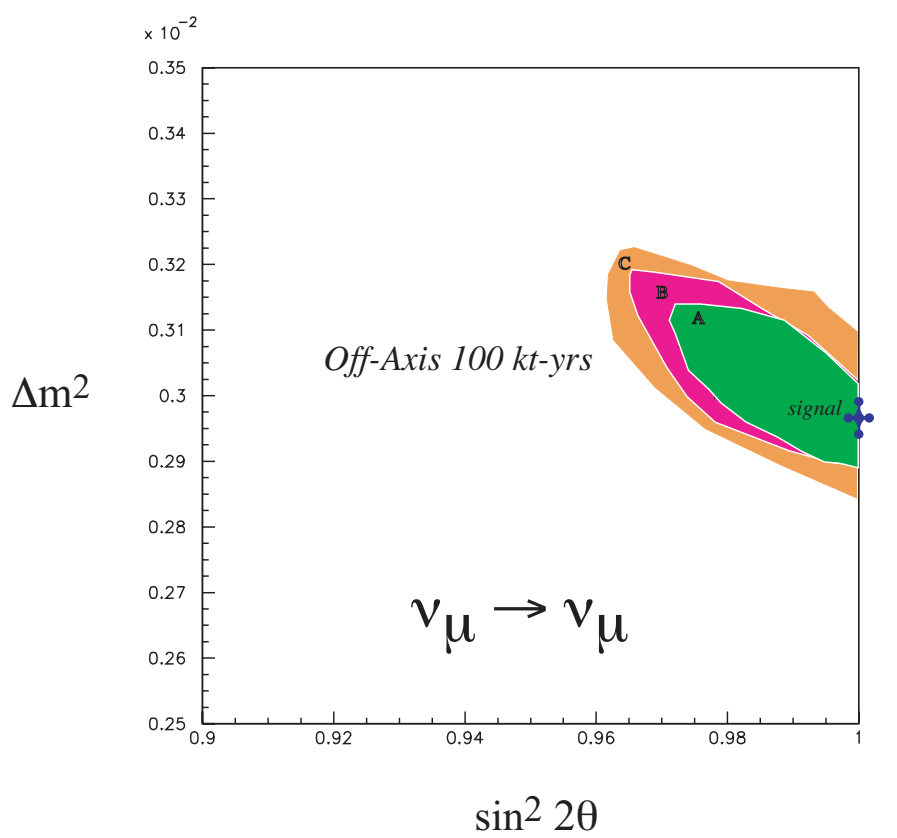

Figure 2. Full set of analyzed errors: (A) Statistical and Detector Resolution, (B) Beam Rate and Spectrum Errors, and (C) Effect of Neutral Current Background. The inner region is Set A, middle region is Sets A and B, outer region is Sets A, $\mathrm{B}$, and $\mathrm{C}$.

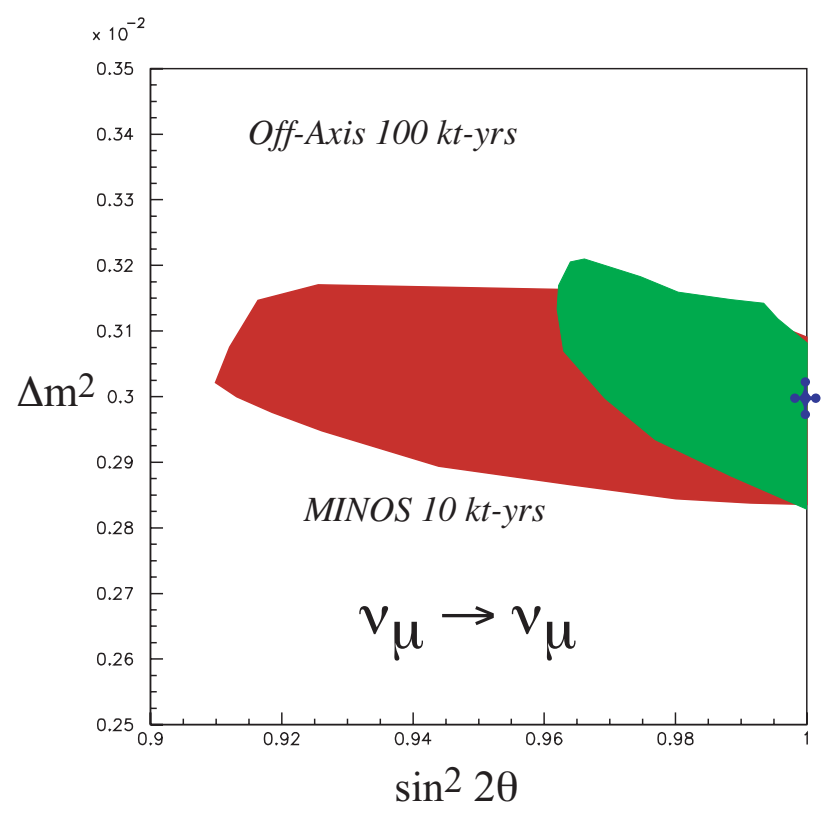

Figure 3. Comparison to MINOS capabilties, all at $90 \% \mathrm{CL}$, for the full set of analyzed errors: (A) Statistical and Detector Resolution, (B) Beam Rate and Spectrum Errors, and (C) Effect of Neutral Current Background. 